\title{
Compressed Sensing based Single Snapshot DoA Estimation for Sparse MIMO Radar Arrays
}

Fabian Roos, Philipp Hügler, Lizette Lorraine Tovar Torres, Christina Knill, Johannes Schlichenmaier, Claudia Vasanelli, Nils Appenrodt, Jürgen Dickmann, and Christian Waldschmidt

(C) 2019 IEEE. Personal use of this material is permitted. Permission from IEEE must be obtained for all other uses, in any current or future media, including reprinting/republishing this material for advertising or promotional purposes, creating new collective works, for resale or redistribution to servers or lists, or reuse of any copyrighted component of this work in other works. 


\title{
Compressed Sensing based Single Snapshot DoA Estimation for Sparse MIMO Radar Arrays
}

\author{
Fabian Roos ${ }^{\# 1}$, Philipp Hügler" ${ }^{\#}$ Lizette Lorraine Tovar Torres ${ }^{\#}$, Christina Knill ${ }^{\#}$, Johannes Schlichenmaier ${ }^{\#}$, \\ Claudia Vasanelli", Nils Appenrodt*, Jürgen Dickmann*, and Christian Waldschmidt ${ }^{\#}$ \\ \# Institute of Microwave Engineering, Ulm University, 89081 Ulm, Germany \\ *Daimler AG, Group Research and Advanced Engineering, 89081 Ulm, Germany \\ ${ }^{1}$ roos@ieee.org
}

\begin{abstract}
The angular resolution of a radar system can be enhanced with an increasing antenna aperture. Instead of using more antenna elements, the distances in the aperture can be increased with a sparse array. To mitigate the high side lobes originating from the sparse array, the missing antenna elements can be reconstructed by means of compressed sensing. In this paper a sparse antenna array with a low side lobe level is determined with a genetic algorithm and a cost function. An investigation is performed what difference in the radar cross section of two targets in the same range-Doppler cell can be achieved. Additionally, instead of considering point targets only, a target vehicle is measured with a $77 \mathrm{GHz}$ MIMO radar.

Keywords - MIMO radar, direction-of-arrival estimation, sparse arrays, compressed sensing, single snapshot, extended targets, automotive radar
\end{abstract}

\section{INTRODUCTION}

One key parameter for driver assistance systems is a high angular resolution [1], which is directly linked to the size of the antenna aperture. In addition, a fast update rate demands the evaluation of single snapshots. To reduce the hardware effort, a multiple-input multiple-output (MIMO) approach is often chosen. The resulting virtual aperture can be further enlarged by increasing the spacings of the virtual antenna elements resulting in a non-uniform array. A well-known approach is a minimum-redundancy array introduced in [2] or sparse arrays in general, which expand the aperture but increase the side lobes.

To counteract the high side lobes of a sparse array and to achieve an improved direction-of-arrival (DoA) estimation, high-resolution algorithms like MUSIC can be chosen. Those subspace-based algorithms rely on the computation of the covariance matrix, for which a single snapshot can lead to increasing errors [3]. Additionally, the knowledge of the number of targets is required, which in the most cases is unknown. This motivates the reconstruction of the missing virtual antenna elements with compressed sensing-based algorithms. This approach requires that a signal is compressible, i.e. that it only contains a limited number of targets. In most cases the automotive environment consists of extended targets like vehicles with a lot of scattering centres [4]. For a chirp-sequence modulated radar [5] targets are separated in different range-Doppler cells. Hence, only a limited number of targets is expected in one cell and the assumption of few targets can be considered valid.
In [6] the increased resolution of a sparse array in contrast to a uniform linear array (ULA) with the same amount of elements is shown. The distance between two close targets with the same radar cross section (RCS) is reduced until they cannot be distinguished by a ULA. Only the sparse array with compressed sensing can separate the closest targets.

The authors of [7] use a prime number-based array setup for the transmit and receive array. In simulation, the advantage of the compressed sensing-based evaluation of two widely separated targets is shown.

In the recent publication of [8] different sparse array realisations are compared with different compressed sensing reconstruction algorithms. Different measurement scenarios with two differently sized corner reflectors are presented.

In this paper an optimised sparse antenna array is designed with a genetic algorithm and boundary conditions given by the used hardware and fabrication process. The missing antenna elements of the sparse array are reconstructed with a compressed sensing algorithm. This algorithms does not require knowledge of the number of targets in the considered scenario. The maximum RCS difference between two targets in the same range-Doppler cell still to be distinguished is investigated. Finally, a measurement of an extended target is presented to verify that the proposed signal processing can cope with multiple targets in the same range-Doppler cell.

\section{Sparse ArRay Design}

A genetic algorithm is applied to determine an optimised sparse array [9] for the existing experimental radar sensor. The virtual aperture of each sparse array realisation is evaluated with the cost function

$$
q=\frac{P}{\mathrm{AFR}},
$$

consisting of the ambiguity-free region (AFR) and a punishment factor $P$. The AFR is the size of the area around boresight in which the ambiguity function [10], [11] is below 0.5 . The additional punishment factor $P$ is the number of the ambiguity function values which are above $-12 \mathrm{~dB}$. This ensures that no high values directly outside of the ambiguity-free region are present and controls the side lobe level in general.

The boundary conditions are mainly defined by the experimental radar system [12], which uses a waveguide 


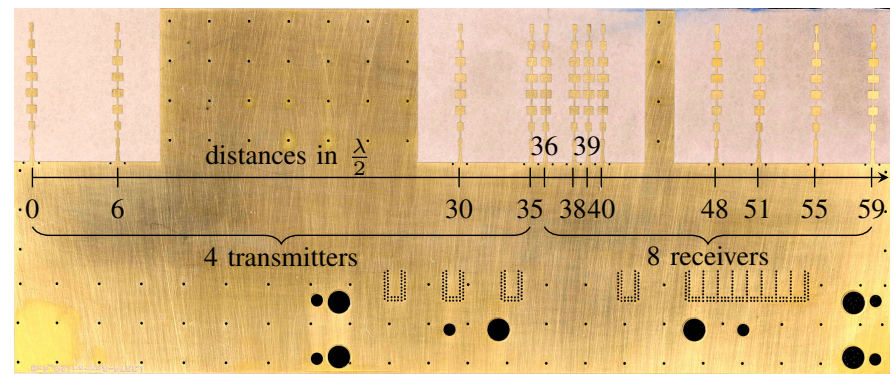

Fig. 1. Fabricated sparse antenna on a PCB with the element distances in multiples of $\frac{\lambda}{2}$ at $76.5 \mathrm{GHz}$.

transition to an exchangeable antenna printed circuit board (PCB). Four transmitters and eight receivers are available in total. As the transmit and receive transitions are placed as a block next to each other, for a simple two-layer fabrication process it is not possible to mix transmit and receive elements. Hence, all the transmit elements are placed on the left side and all receive elements on the right side. The maximum PCB size is set to $120 \mathrm{~mm}$ as a trade-off between a sufficient angular resolution and too long feeding lines leading to large losses. For the Fourier transform-based reconstruction algorithm, a virtual aperture grid with equidistant spacing is ideal, hence, only array element positions in multiples of $\frac{\lambda}{2}$ are allowed.

The optimised sparse array shown in Fig. 1 is fabricated on a Rogers 3003 substrate with a thickness of $127 \mu \mathrm{m}$. In contrast to a ULA with 32 elements, the virtual aperture is nearly doubled from $15.5 \lambda$ to $29 \lambda$. Using the Rayleigh criterion this results in an angular resolution of $2.4^{\circ}$ instead of $4.5^{\circ}$.

To characterise the sparse antenna, the ambiguity function is measured and shown in Fig. 2. As the minimum distance in the virtual aperture is set to $\frac{\lambda}{2}$, no grating lobes are present. The antenna array consists of single patch antennae limiting the maximum field of view to roughly $\pm 70^{\circ}$ in azimuth direction. This is noticeable at the edges of the ambiguity function where

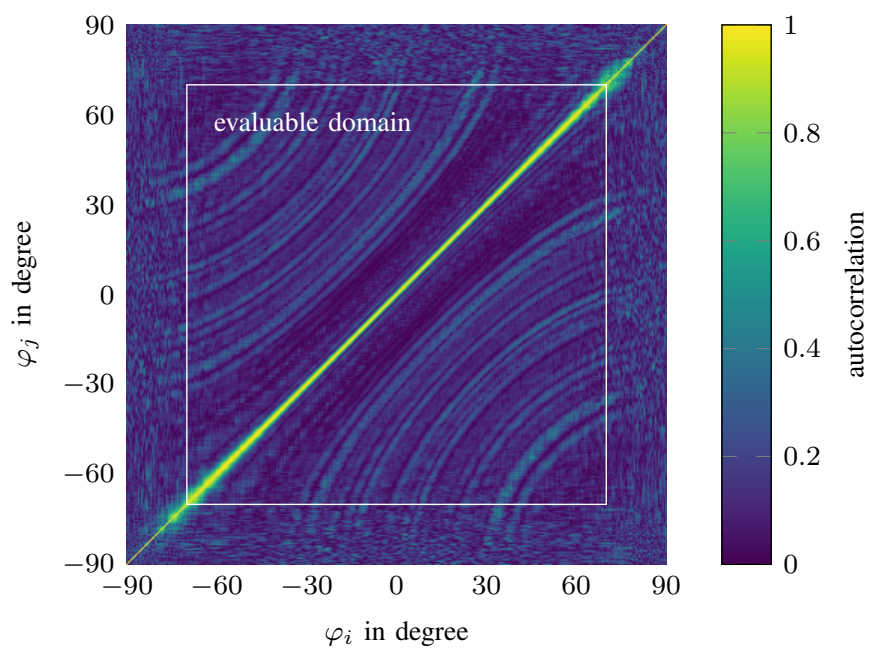

Fig. 2. Measured ambiguity function of the sparse antenna array shown in Fig. 1. the diagram is blurred. Values close to 0.5 are only present at the resulting field of view edges.

\section{COMPRESSED SENSING-BASED RECONSTRUCTION}

For each channel a range-Doppler spectrum is obtained, which is combined to a single spectrum by taking the mean of the absolute values, i.e. non-coherently integrated. A DoA estimation is performed for the identified target cells. The missing elements of the sparse virtual array lead to high side lobes preventing a reliable detection of weak targets. Therefore, those elements are reconstructed with the iterative method with adaptive threshold (IMAT) [13] algorithm.

The missing elements are represented by zeros in the steering vector of the virtual array. To achieve a smoother spectrum, this steering vector is zero-padded to 256 values. The fundamental concept of the IMAT algorithm starts with the calculation of the Fourier transform of the sparse steering vector. The highest peak in the spectrum (---) shown in Fig. 3 is considered to be a valid target. Hence, the maximum of the spectrum is chosen as the first threshold thr(1). All values above the current threshold $\operatorname{thr}(k)$, which is consecutively lowered in the next iterations, are used to estimate the missing elements. Thus, there is no assumption of the number of present targets. As every frequency above the threshold is intensified, the crucial part is to stop before the threshold is lowered into noise. This is achieved as described in [14] by calculating the mean of the sparse spectrum. Although the noise level is overestimated, an additional safety margin of $3 \mathrm{~dB}$ is added. As already mentioned, the missing elements lead to high artefacts in the spectrum. With each iteration the missing elements are better approximated, and the artefacts are suppressed. This enables the detection of weak targets, whose peaks are not lowered, but staying nearly constant. In Fig. 3, the artefacts in the reconstructed spectrum (-) are reduced after $k=5$ iterations, but the peak (o) of the real target at nearly $0^{\circ}$ stays constant. In the following iterations the threshold is further lowered, and the weak target peak is enhanced.

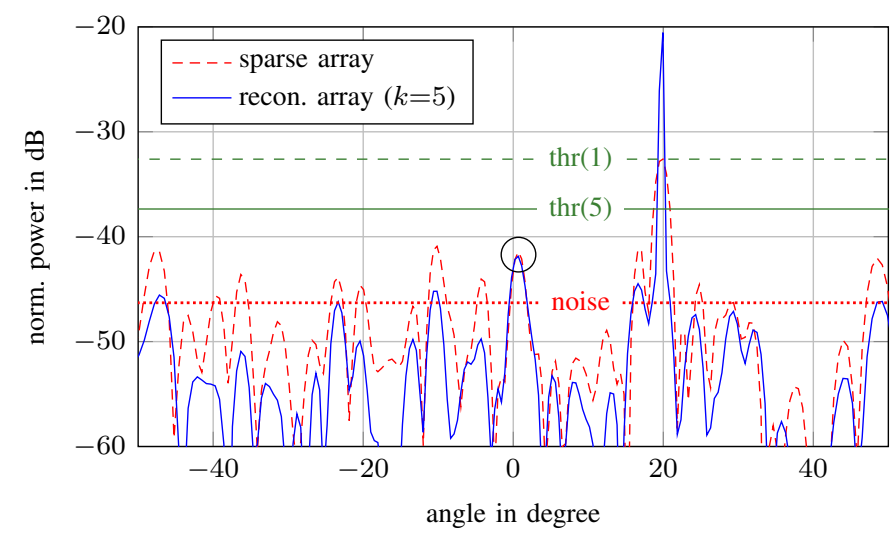

Fig. 3. The IMAT algorithm calculates the spectrum of the sparse steering vector (---) and uses the maximum as a first threshold thr(1). All frequencies above the threshold are used for the reconstruction of the missing elements $(-)$. The stopping level is determined with a rough noise level estimation (..........) and an added safety margin. One weak target is present (O). 
For reconstructing the missing virtual elements $k=10$ iterations are used. This leads to a high artefact suppression and to a high signal-to-noise ratio. The required iteration number depends on the amount of missing data as shown in [15]. Even with a reduced iteration number a sufficient reconstruction is feasible, as further iterations only lead to minor improvements.

\section{Measurement Results}

To verify the performance of the fabricated sparse antenna, measurements are performed with an experimental $77 \mathrm{GHz}$ MIMO radar system [12]. The modulation parameters are listed in Table 1.

\section{A. Achievable Difference in RCS}

A challenge for a radar is the detection of two targets with substantially different radar cross sections, especially if they are situated in the same range-Doppler cell. In an automotive environment, a pedestrian next to a vehicle can lead to a very large difference. Hence, an investigation in an anechoic chamber is performed, what difference in RCS can be resolved if the targets are in the same cell. In this scenario point targets realised with corner reflectors are considered.

In the first measurement setup a corner reflector with an RCS of $-9.3 \mathrm{dBsm}$ is placed at $0^{\circ}$. A second corner reflector with the same RCS value is placed at $20^{\circ}$ and is getting replaced with reflectors with successively higher RCS values until the first reflector cannot be detected anymore. A reliable detection in several measurements was possible with an $0.5 \mathrm{dBsm}$-corner reflector leading to a difference in RCS of $9.8 \mathrm{~dB}$. A measurement example of this setup is shown in Fig. 4. After reconstruction (-), the difference between the weak target and the first noise peak is more than $10 \mathrm{~dB}$ leading to a reliable detection. The evaluation of the sparse array without reconstruction (---) does not allow for a detection of the weak target. In fact, the artefact peak at $-10^{\circ}$ has an $0.5 \mathrm{~dB}$ higher level than the real target.

In the second setup a corner reflector with an RCS of $0.5 \mathrm{dBsm}$ is placed next to an corner reflector of $9.4 \mathrm{dBsm}$ leading to a difference of $8.9 \mathrm{~dB}$. In successive measurements the angle between the corner reflectors is increased until a reliable detection is possible once again. The minimum angle achieved corresponds to a difference in the direction-or-arrival estimation of only $2.6^{\circ}$ which is close to the theoretical limit of $2.4^{\circ}$. Again, only after the successful reconstruction $(-)$, the two targets can be distinguished as shown in Fig. 5.

Table 1. Specifications of Radar Parameters

\begin{tabular}{lr} 
Parameter & Value \\
\hline carrier frequency $f_{\mathrm{c}}$ & $76.5 \mathrm{GHz}$ \\
bandwidth $B$ & $2 \mathrm{GHz}$ \\
chirp duration $T_{\mathrm{c}}$ & $50 \mu \mathrm{s}$ \\
chirp repetition time $T_{\mathrm{r}}$ & $60 \mu \mathrm{s}$ \\
sampling frequency $f_{\mathrm{s}}$ & $20 \mathrm{MHz}$ \\
number of chirps $L$ & 256
\end{tabular}

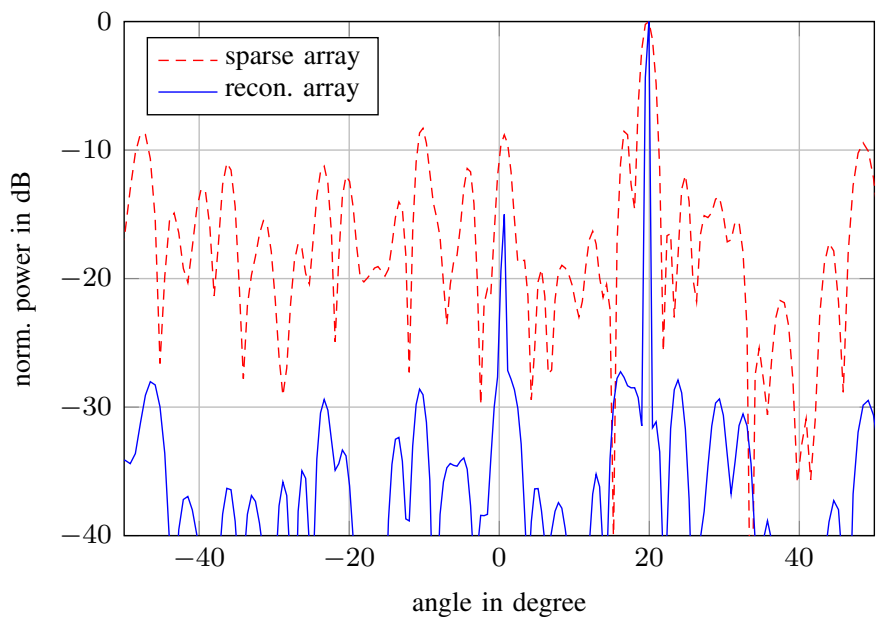

Fig. 4. Measurement of two targets at $0^{\circ}$ and $20^{\circ}$ with an RCS difference of $9.8 \mathrm{~dB}$. Only after reconstruction (-) of the missing elements in the sparse array, the weak target can be distinguished from noise.

\section{B. Extended Target Detection}

As an extended target a vehicle is measured. It is parked in front of the radar sensor so that both the front and the long side are visible. The range-Doppler plot of the vehicle is shown in Fig. 6 (a), inlcuding the associated range-Doppler cells. For every range-Doppler cell corresponding to the vehicle the compressed sensing-based reconstruction is performed.

An exemplary DoA estimation result of a single cell consisting of three scattering centres is shown in Fig. 6 (b). To differentiate between noise and possible targets a rough estimation of the noise level (---) is calculated using the mean of the reconstructed spectrum (-). A safety margin of $16 \mathrm{~dB}$ is added to the overestimated noise level resulting in the threshold (-) level. Every spectral peak which lies above the threshold and declines in amplitude around the peak of at least $3 \mathrm{~dB}$ is considered a valid scattering centre.

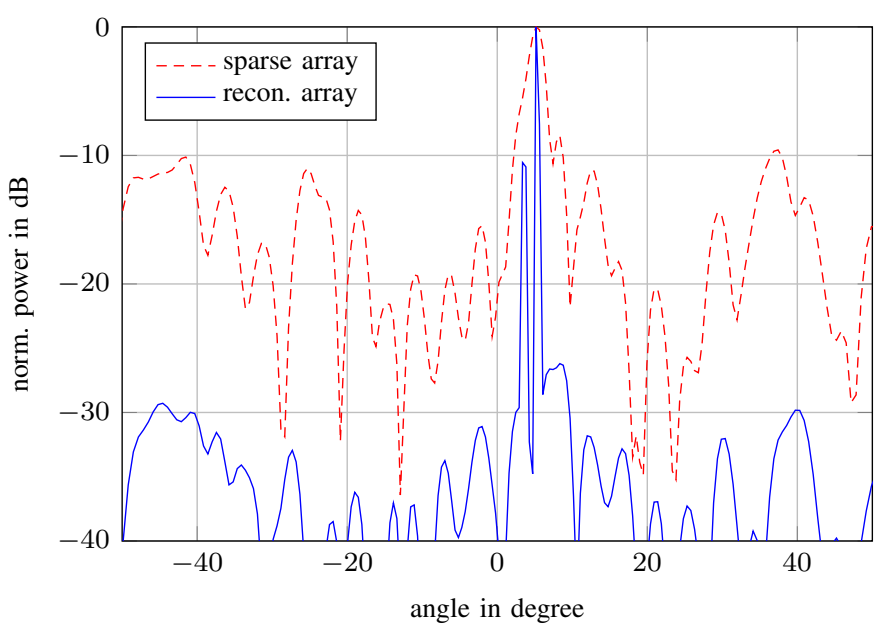

Fig. 5. Measurement of two close targets separated with only $2.6^{\circ}$ and an RCS difference of $8.9 \mathrm{~dB}$. Only after the compressed sensing-based reconstruction (- ${ }^{-}$, the two targets can be separated. 


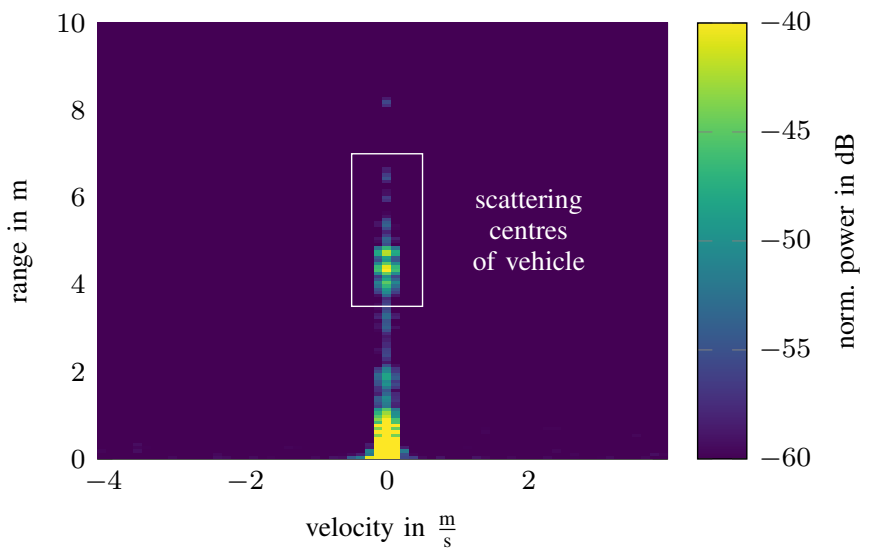

(a) Different scattering centres are present in the range from $3.7 \mathrm{~m}$ to $6.5 \mathrm{~m}$.

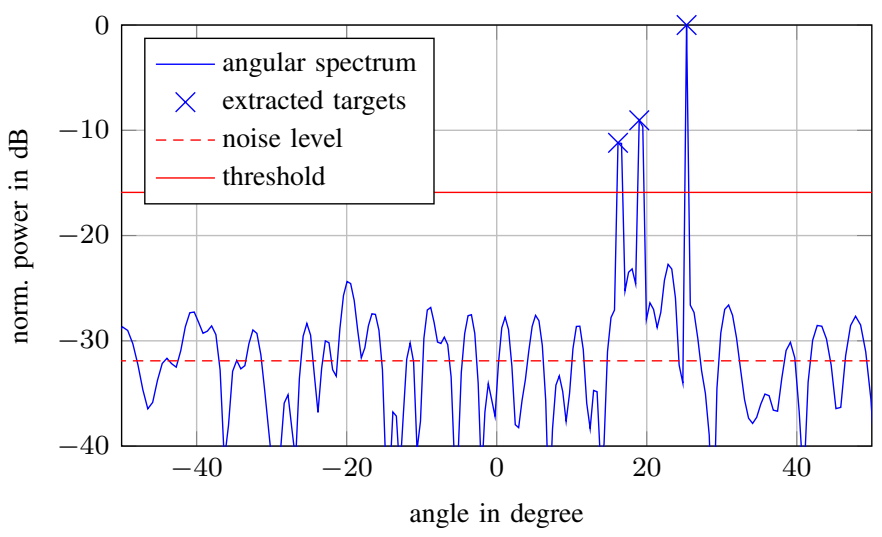

(b) In the reconstructed spectrum of a single range-Doppler cell every prominent peak above the threshold $(-)$ is considered as a scattering centre $(X)$.

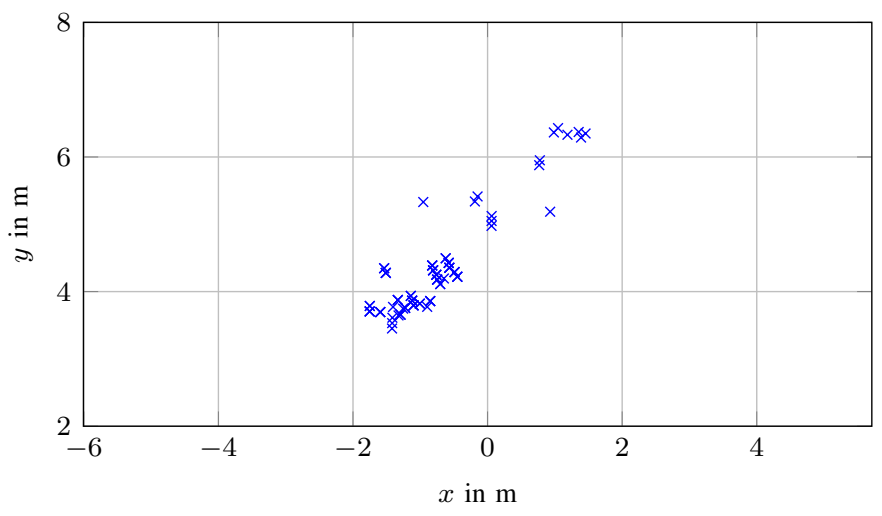

(c) The DoA estimation of all range-Doppler cells results in the $x$-y-plot.

Fig. 6. Measurement of a stationary target vehicle with several different scattering centres in (a). For an exemplary range-Doppler cell the direction-of-arrival estimation is shown in (b). This results in the $x-y$-plot shown in (c)

The resulting $x$-y-diagram of the measured vehicle is depicted in Fig. 6 (c). The contour of the long side of the vehicle is partly discontinued, as only some prominent scattering centres as the wheel houses and the bumper are present. However, the available contour points enable, e.g., an orientation estimation [16].

\section{CONCLUSION}

The angular resolution of a radar is often enhanced with a sparse array. The omitted antenna elements induce high side lobes limiting the detection of weak targets in the same range-Doppler cell. In this paper, an optimised sparse antenna array is determined with the help of a genetic algorithm and an ambiguity function-based cost function. The missing elements are reconstructed with a compressed sensing algorithm lowering the artefacts and enabling the detection of weak targets. The algorithm is applied to single snapshots and does not require knowledge of the number of targets. An investigation is performed what maximum difference in the RCS of two targets in the same range-Doppler cell can reliably be detected. Measurement results show that a difference of nearly $10 \mathrm{~dB}$ can still be resolved. As the automotive environment consists of extended targets, a target vehicle is measured with the sparse array to prove that extended targets can be resolved.

\section{REFERENCES}

[1] J. Dickmann et al., "”Automotive Radar the Key Technology for Autonomous Driving: From Detection and Ranging to Environmental Understanding"," in IEEE Radar Conference, May 2016, pp. 1-6.

[2] A. T. Moffet, "Minimum-Redundancy Linear Arrays," IEEE Trans. Antennas Propag., vol. 16, no. 2, pp. 172-175, Mar. 1968.

[3] I. Ziskind and M. Wax, "Maximum Likelihood Localization of Multiple Sources by Alternating Projection,' IEEE Trans. Acoust., Speech, Signal Process., vol. 36, no. 10, pp. 1553-1560, Oct. 1988.

[4] M. Andres et al., "Analysis of Automobile Scattering Center Locations by SAR Measurements," in Radar Conference, May 2011, pp. 109-112.

[5] V. Winkler, "Range Doppler Detection for automotive FMCW Radars," in European Radar Conference, Oct. 2007, pp. 166-169.

[6] F. Belfiori et al., "Digital Beam Forming and Compressive Sensing Based DOA Estimation in MIMO Arrays," in 8th European Radar Conference, Oct. 2011, pp. 285-288.

[7] A. Kirschner et al., "Enhanced Angular Resolution and Ambiguity Suppression for MIMO Radar Systems by Compressed Sensing Techniques," in European Radar Conference (EuRAD), Oct. 2013.

[8] A. Correas-Serrano and M. A. González-Huici, "Experimental Evaluation of Compressive Sensing for DoA Estimation in Automotive Radar," in International Radar Symposium, Jun. 2018, pp. 1-10.

[9] R. L. Haupt, "Thinned Arrays Using Genetic Algorithms," IEEE Transactions on Antennas and Propagation, vol. 42, no. 7, pp. 993-999, Jul. 1994.

[10] M. Erić et al., "Ambiguity characterization of arbitrary antenna array: type i ambiguity," in IEEE 5th International Symposium on Spread Spectrum Techniques and Applications, vol. 2, Sep. 1998, pp. 399-403.

[11] C. Vasanelli et al., "Assessment of a Millimeter-Wave Antenna System for MIMO Radar Applications," IEEE Antennas and Wireless Propagation Letters, vol. 16, pp. 1261-1264, 2017.

[12] P. Hügler et al., "Radar Taking Off: New Capabilities for UAVs," IEEE Microwave Magazine, vol. 19, no. 7, pp. 43-53, Nov. 2018.

[13] F. Marvasti et al., "A Unified Approach to Sparse Signal Processing," EURASIP Journal on Advances in Signal Processing, 2012.

[14] F. Roos et al., "Data Rate Reduction for Chirp-Sequence based Automotive Radars using Compressed Sensing," in German Microwave Conference (GeMiC), Mar. 2018, pp. 347-350.

[15] F. Roos et al., "Effort Considerations of Compressed Sensing for Automotive Radar," in Radio and Wireless Symposium (RWS), 2019.

[16] F. Roos et al., "Reliable Orientation Estimation of Vehicles in High-Resolution Radar Images," IEEE Trans. Microw. Theory Techn., vol. 64, no. 9, pp. 2986-2993, Sep. 2016. 